\title{
Prevalencia de enfermedades dentales en gatos (felis catus) de los distritos del cono norte de Lima.
}

\author{
Prevalence of dental disease in cats (feliz catus) in the northern districts of lima
}

Ricardo Grandez ${ }^{1}$, Heidi Guerrero ${ }^{1}$

RESUMEN

Objetivo: Determinar la prevalencia de las patologías dentales de los gatos (Felis catus) del Cono Norte de Lima atendidos en la Clínica Veterinaria de la Universidad Peruana Cayetano Heredia. Material y métodos: Se evaluaron 200 felinos de 1 a 16 años de edad, sin distinción de raza y sexo. Las variables evaluadas fueron: edad ( $\geq 1$ a $<5, \geq 5$ a $<9, \geq 9$ años), tipo de alimentación (balanceada, mixta sin hueso y mixta con hueso) y localización de la patología dental. Resultados: Se determinó que el 95,5\% de los felinos presentaron algún grado de enfermedad periodontal, y los dientes más afectados por la enfermedad periodontal fueron los dientes caninos y el cuarto premolar superior. Los felinos que consumieron el alimento mixto con hueso tuvieron una mayor frecuencia de enfermedad periodontal avanzada. La fractura dental fue la única patología que mostró una asociación con la edad. El 31\% de los felinos presentaron fractura dental (caninos), siendo los animales más afectados los que consumieron alimento mixto con hueso. Otras patologías observadas fueron el Síndrome Gingivitis-Estomatitis y masas orales. El tipo de alimentación y el sexo no estuvo estadísticamente asociado a la presentación de las enfermedades dentales. Conclusiones: Los resultados permiten sustentar que la enfermedad periodontal y la fractura dental son patologías comunes en los gatos del Cono Norte de Lima.

PALABRAS CLAVE: Patologías dentales, gatos, enfermedad periodontal, alimento.

\section{SUMMARY}

Objectives: The study aimed to determine the prevalence of dental diseases in cats (Felis catus) of the northern districts of Lima that were patients of the Clínica Veterinaria of the Universidad Peruana Cayetano Heredia. Material and methods: Two hundred cats, from 1 to 16 years of age, and without distinction of breed and sex were evaluated. The variables under evaluation were: age ( $\geq 1$ to $<5, \geq 5$ to $<9, \geq 9$ years), type of diet (balanced, mixed without bone, mixed with bone), and location of the dental pathology. Results: The results showed that 95,5\% of examined cats presented periodontal disease, where the canine teeth and the fourth upper premolar were the most affected. Those that consumed mixed feed with bone had higher frequency of advanced periodontal disease. Dental fracture (canine teeth) was found in $31 \%$ of the cats, being most affected those that consumed mixed food with bone. Also, dental fracture was the only dental pathology that showed an association with age. Other pathologies were the Gingivitis-Stomatitis Syndrome and oral masses. The type of food and sex were not statistically associated with the presentation of dental diseases. Conclusions: The results allow to sustain that periodontal disease and dental fractures are common pathologies in cats in the northern districts of Lima.

KEY WORDS: dental pathologies, cats, periodontal disease, food.

Facultad e Medicina Veterinaria y Zootecnia - Universidad Peruana Cayetano Heredia 


\section{INTRODUCCIÓN}

La enfermedad dental es uno de los problemas más comunes que afecta a los animales domésticos $(1,2)$, siendo la odontología felina una disciplina relativamente nueva dentro del campo de la odontología veterinaria. El primer programa exclusivo de educación dental felina fue patrocinado por la Asociación Americana de Profesionales en Felinos, en 1988 (3).

Se ha descrito que el primer tratamiento dental para los gatos debe realizarse entre 1 y 2 años de edad, y luego a intervalos de 6 a 12 meses; aunque se han reportado tratamientos en edades más tempranas, debido a la gingivitis (3).El número de dientes permanentes del gato es 30 , siendo la fórmula dentaria: $2 \times(\mathrm{I} / 3$, $\mathrm{C} 1 / 1, \mathrm{P} 3 / 2, \mathrm{M} 1 / 1)=30$ (4). Las patologías dentales en los gatos se agrupan en las siguientes categorías: enfermedad periodontal, resorción dental (FORL), fractura dental, Síndrome Gingivitis-Estomatitis y masas orales $(5,6)$.

El $85 \%$ de los gatos mayores de 4 años presentan patologías dentales $(7,8)$, siendo la enfermedad periodontal la más habitual $(9,10,11)$. Esta patología puede llegar a establecer una condición debilitante que progresa de gingivitis a periodontitis; que conlleva a la pérdida de piezas dentales $(12,13)$.

La periodontitis se caracteriza por producir signos clínicos locales, tales como congestión de encías, depósito de placa y cálculo dental, sangrado, retracción gingival, formación de bolsa periodontal, movilidad dentaria y pérdidas dentarias $(14,15)$. En algunos casos, se presentan efectos sistémicos como consecuencia del ingreso de gérmenes, pudiendo producir endocarditis bacterianas, artritis sépticas, lesiones renales y un desmejoramiento del estado general del animal $(16,17)$.

La fractura dental, de una parte o de toda la corona, es más frecuente en los dientes caninos $(18,19)$; en tanto que el Síndrome Gingivitis- Estomatitis, de etiología desconocida y de baja prevalencia (20), se caracteriza por una inflamación crónica severa de la gingiva, mucosa alveolar y mucosa gloso palatina $(11,21)$.

Otra patología frecuente es la resorción ósea $(22,23)$, también llamada lesión de la línea cervical o del cuello del diente felino. Tiende a iniciarse en el margen gingival o subgingival y progresa en dirección apical o coronal. Estas lesiones son discretas en su primera etapa, por lo que se requiere emplear el diagnóstico radiográfico para su detección $(11,24,25)$.

Los felinos pueden presentar diversos tipos de neoplasias orales $(26,27,28)$. El carcinoma de células escamosas es la neoplasia oral de mayor ocurrencia, y normalmente afecta las encías y la lengua, con un mal pronóstico $(29,30)$. Otra neoplasia de importancia, aunque menos común, es el fibrosarcoma, que es localmente invasivo y de mal pronóstico. Ambas neoplasias se presentan usualmente en animales de 10 a13 años, pero también se ha reportado en gatos de 1 a 22 años de edad $(31,32)$. Las neoplasias orales pueden afectar al tejido blando, al hueso y a las estructuras propias del diente $(26,31)$.

El objetivo de la presente investigación fue documentar la prevalencia de las enfermedades dentales de los gatos de los distritos del Cono Norte de Lima, a fin de ampliar el conocimiento sobre los principales problemas orales que afectan a esta población.

\section{MATERIAL Y MÉTODOS}

El presente estudio se realizó en el marco de la campaña "Descarte de Enfermedades Dentales en Felinos", entre noviembre de 2006 y abril de 2007, implementada por la Clínica Veterinaria Cayetano Heredia de la Universidad Peruana Cayetano Heredia, en el distrito de San Martín de Porres, Lima. Se trabajó con 200 ejemplares felinos, sexualmente maduros, de 1 a 16 años de edad, provenientes de los distritos del Cono Norte de Lima (San Martín de Porres, Los Olivos, Independencia y Puente Piedra).

La distribución de los 200 felinos de acuerdo al grupo racial fue: 20 Persas, 7 Siameses, 1 Birmano y 1 Angora Turco, siendo el resto $(\mathrm{n}=171)$ de tipo mestizo. Asimismo, 116 (58\%) fueron hembras y 84 (42\%) machos; en tanto que 89 (44,5\%) consumían alimento balanceado, 73 (36,5\%) alimento mixto sin hueso, y 38 (19\%) alimento mixto con hueso. Ninguno presentó alteraciones clínicas durante el examen clínico general.

A cada animal se le realizó un examen clínico general y un examen odontológico, anotándose la reseña y la anamnesis. Se registró la edad, sexo, peso y tipo de alimentación, se tomaron las constantes vitales, y se les realizó el examen físico clínico general. Para el registro de estos datos se elaboró una 

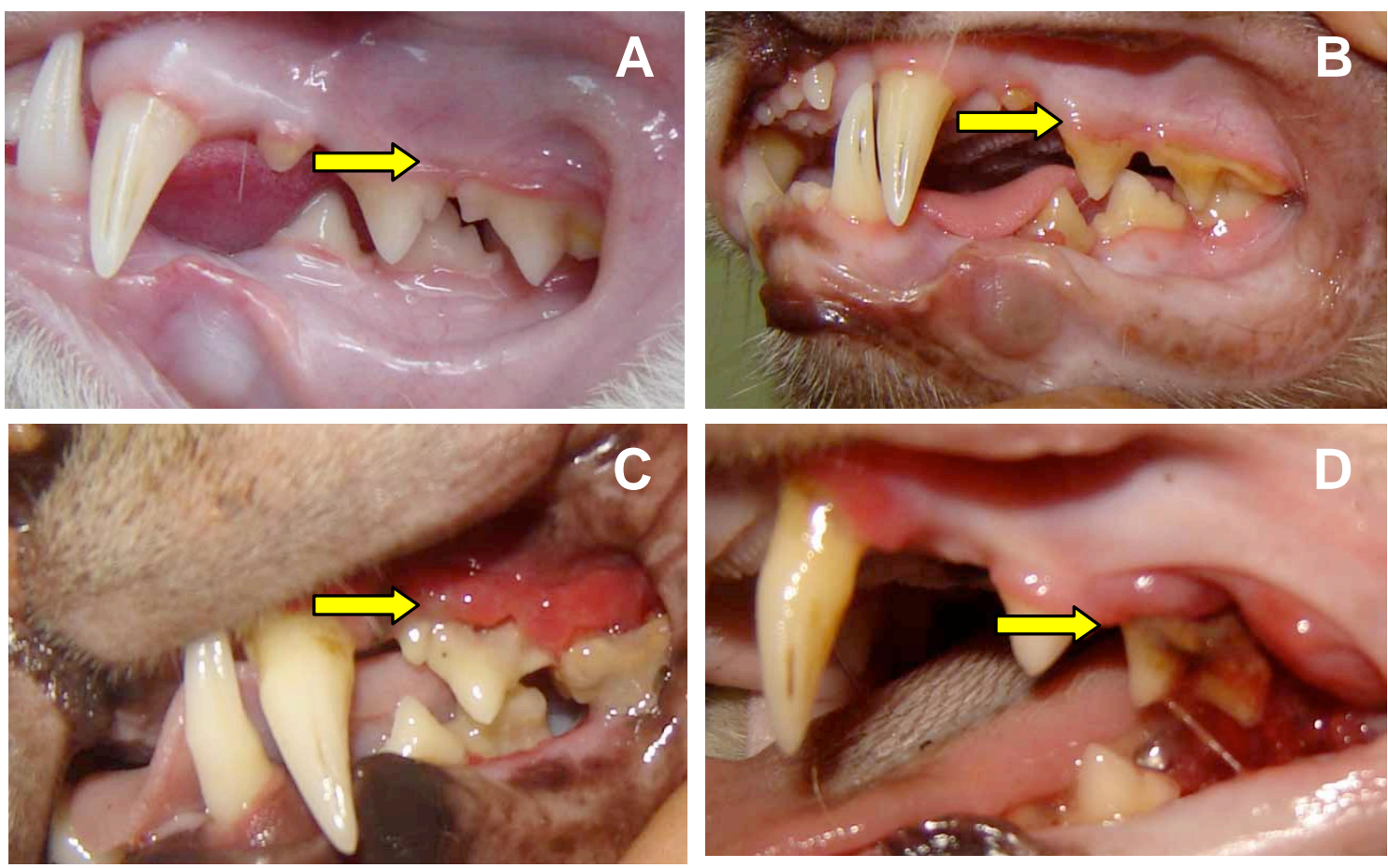

Figura 1.Enfermedades dentales en gatos (Felis catus) de los distritos del Cono Norte de Lima; grados de enfermedad periodontal según la clasificación de Holmstrom (2000b). (A) Grado I: enrojecimiento de la encía en la cresta gingival y pequeña cantidad de placa; (B) Grado II: similar al I con incremento de la inflamación y aparición de cálculo dental; (C) Grado III: gingivitis con inicio de la formación de bolsas gingivales e incremento de la cantidad de placa y cálculo; (D) Grado IV: inflamación grave con formación de bolsas profundas, recesión gingival, pérdida de hueso y movilidad dental.
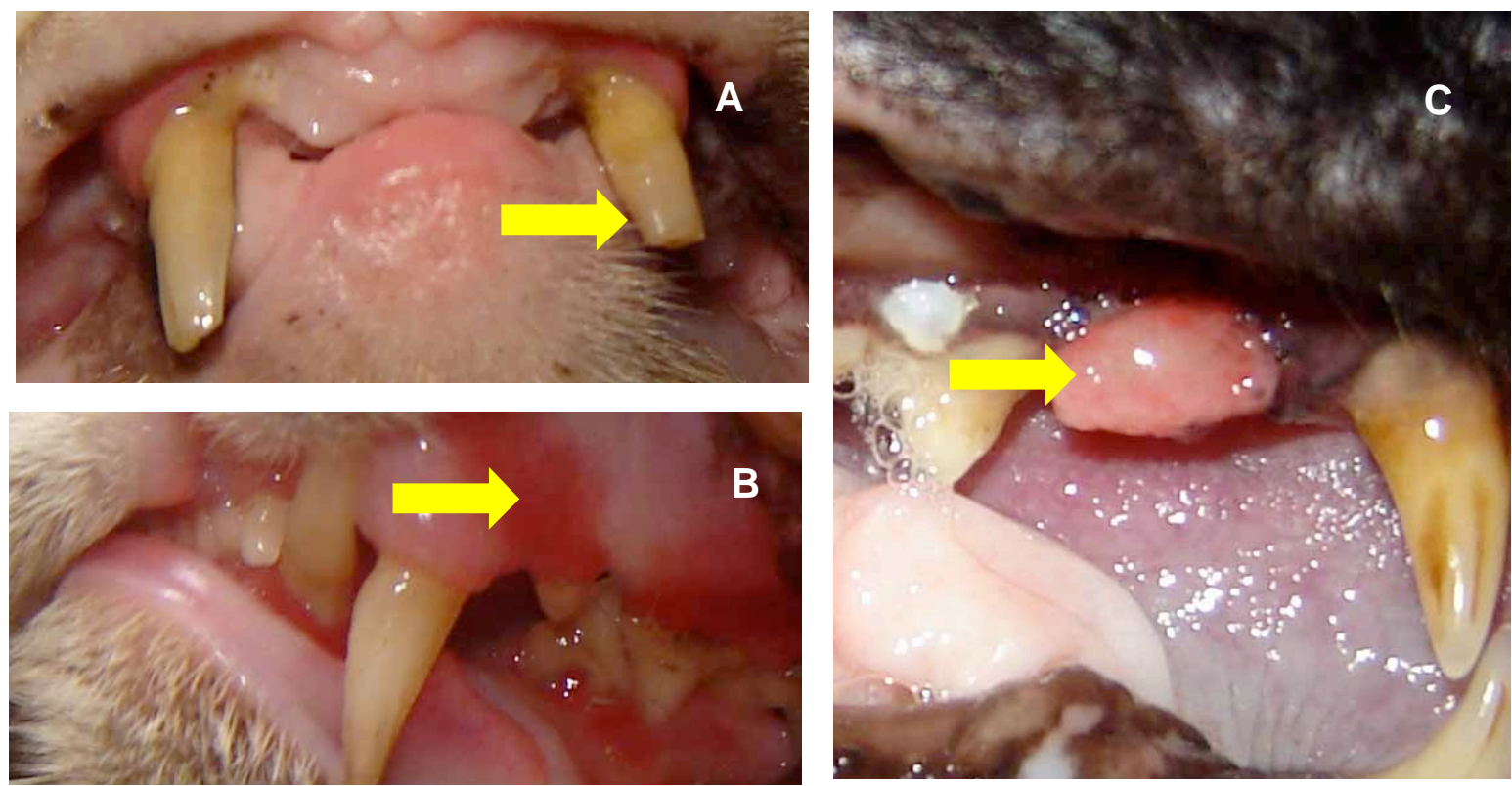

Figura 2. Enfermedades dentales en felinos en los distritos del Cono Norte de Lima. (A) Fracturas dentales. (B) Síndrome Gingivitis-Estomatitis. (C) Masa oral. 
Tabla 1. Prevalencia de enfermedades dentales en felinos, de acuerdo a la edad, en los distritos del Cono Norte de Lima

\begin{tabular}{|c|c|c|c|c|}
\hline Enfermedad & Edad (años) & $\mathrm{n}$ & Positivos & Porcentaje \\
\hline \multirow{4}{*}{ Enfermedad Periodontal } & $\geq 1 \mathrm{a}<5$ & 134 & 125 & $93,2^{\mathrm{a}}$ \\
\hline & $\geq 5 \mathrm{a}<9$ & 48 & 48 & $100,0^{\mathrm{a}}$ \\
\hline & $\geq 9$ & 18 & 18 & $100,0^{\mathrm{a}}$ \\
\hline & TOTAL & 200 & 191 & 95,5 \\
\hline \multirow{4}{*}{$\begin{array}{c}\text { Síndrome Gingivitis- } \\
\text { Estomatitis }\end{array}$} & $\geq 1$ a $<5$ & 134 & 2 & $1,5^{\mathrm{a}}$ \\
\hline & $\geq 5 \mathrm{a}<9$ & 48 & 1 & $2,0^{\mathrm{a}}$ \\
\hline & $\geq 9$ & 18 & 0 & $0,0^{\mathrm{a}}$ \\
\hline & TOTAL & 200 & 3 & 1,5 \\
\hline \multirow{3}{*}{$\begin{array}{c}\text { Fractura } \\
\text { Dental }\end{array}$} & $\geq 1 \mathrm{a}<5$ & 134 & 24 & $17,9^{\mathrm{a}}$ \\
\hline & $\geq 5 \mathrm{a}<9$ & 48 & 26 & $54,2^{\mathrm{b}}$ \\
\hline & $\geq 9$ & 18 & 12 & $66,7^{\mathrm{b}}$ \\
\hline \multirow{5}{*}{$\begin{array}{l}\text { Masas } \\
\text { Orales }\end{array}$} & TOTAL & 200 & 61 & 31,0 \\
\hline & $\geq 1 \mathrm{a}<5$ & 134 & 0 & $0,0^{\mathrm{a}}$ \\
\hline & $\geq 5 \mathrm{a}<9$ & 48 & 1 & $2,1^{\mathrm{a}}$ \\
\hline & $\geq 9$ & 18 & 2 & $11,1^{\text {a }}$ \\
\hline & TOTAL & 200 & 3 & 1,5 \\
\hline
\end{tabular}

a,b Letras diferentes dentro de cada patología indican asociación estadística $(\mathrm{p}<0,05)$

Tabla 2. Distribución entre los grados de enfermedad periodontal de acuerdo a la edad en felinos del Cono Norte de Lima

\begin{tabular}{|c|c|c|c|c|c|c|}
\hline & \multicolumn{5}{|c|}{ Grados } & \multirow{2}{*}{ Total } \\
\hline & 0 & I & II & III & IV & \\
\hline \multicolumn{7}{|l|}{ Edad (años) } \\
\hline$\geq 1 \mathrm{a}<5$ & 7.5 & 57,7 & 35,0 & 0 & 0 & 100,0 \\
\hline$\geq 5 \mathrm{a}<9$ & 0 & 21,0 & 54,0 & 14,6 & 10,4 & 100,0 \\
\hline$\geq 9$ & 0 & 0 & 20,1 & 37,2 & 42,7 & 100,0 \\
\hline \multicolumn{7}{|l|}{ Alimentación } \\
\hline Balanceada & 6,7 & 41,1 & 42,2 & 3,3 & 6,7 & 100,0 \\
\hline Mixta sin hueso & 4,2 & 37,5 & 38,9 & 15,3 & 4,2 & 100,0 \\
\hline Mixta con hueso & 0 & 37,8 & 29,7 & 16,2 & 16,2 & 100,0 \\
\hline
\end{tabular}

Ficha Clínica Dental Felina. La exploración oral se hizo siguiendo estándares de evaluación odontológica (33), empleándose el explorador, la sonda y el espejo periodontal, y anotándose las alteraciones de cada diente en la Ficha de odontograma. A los animales agresivos se les administró clorhidrato de ketamina $(10-25 \mathrm{mg} / \mathrm{kg})$ y xylacina $(1,1 \mathrm{mg} / \mathrm{kg})$, vía i.m. (34).

El tamaño muestral se determinó mediante la fórmula de cálculo de tamaño de muestra para comprobación de una proporción (35), con un coeficiente de confianza al 95\%, una probabilidad de 0,85 (7), y un error mínimo posible de 0,05 ; dando como resultado una población de 196 animales.

Para el análisis estadístico, los animales se distribuyeron en tres grupos etáreos $(\geq 1 \mathrm{y}<5, \geq 5 \mathrm{y}<9$, $y \geq 9$ años), en base a trabajos previos de (36); sexo (macho, hembra) y tipo de alimentación (balanceada, mixta sin hueso, mixta con hueso), considerando como alimento mixto la combinación de alimento balanceado y casero. Los datos se evaluaron mediante la prueba de chi-cuadrado, empleando un nivel de confianza de $95 \%$ con el programa SPSS 13,0, para determinar si los factores evaluados presentaban asociación con las alteraciones dentales. Asimismo, se determinó la estadística descriptiva de las patologías encontradas.

Los casos de enfermedad periodontal se clasificaron de acuerdo a lo descrito por Holmstrom (37) (Figura 1). En las fracturas dentales, Síndrome GingivitisEstomatitis y masas orales se estableció el diagnóstico 
de acuerdo a las características anátomopatologicas descritas por diversos autores $(18,21,27)$ (Figura 2).

\section{RESULTADOS}

E1 95,5\% de los gatos ( $\mathrm{n}=191$ ) presentó enfermedad periodontal (95,5\%). Además, aparte de esta patología, presentaron fractura dental $(31,0 \%)$, Síndrome Gingivitis-Estomatitis $(1,5 \%)$ y masas orales $(1,5 \%)$. La fractura dental fue la única patología que mostró una asociación con la edad (Tabla 1), siendo los dientes caninos los únicos que presentaron fractura dental. No se encontró asociación significativa entre alguna otra patología con el sexo o el tipo de alimentación, aunque se notó una tendencia hacia un mayor grado de severidad con el consumo de alimentos más groseros.

La enfermedad periodontal constituyó la enfermedad oral más prevalente en los felinos bajo estudio, siendo las piezas dentales más afectadas el cuarto premolar superior $(83,3 \%)$, los caninos superiores (53\%) y los caninos inferiores (38,3\%). La enfermedad periodontal mostró un incremento en relación a los grados de severidad con relación a la edad. En la tabla 2 se aprecia una mayor frecuencia de grados I y II en el grupo de $\geq 1$ y $<5$ años, de grado II en la edad de $\geq 5$ y $<9$ años, y de grados III y IV en animales $\geq 9$ años de edad.

\section{DISCUSIÓN}

El estudio estuvo dirigido a establecer la prevalencia de patologías dentales en felinos del Cono Norte de Lima. Los resultados globales indican que el 95,5\% de los animales presentó algún tipo de patología dental; frecuencia mayor a la observada en otros estudios 87,5\% (36); 85\%(7); y 90\%(38). Asimismo, los resultados referidos a enfermedad periodontal $(95,5 \%)$ son mayores a lo reportado por otros autores: $70 \%$ (39), $73 \%$ (36), y $90 \%$ (38).

En el caso de los estudios arriba mencionados la alimentación fue en base a alimento balanceado comercial seco (granulado), húmedo (enlatado) o mixto (balaceado y casero). En ninguno de ellos se consideró el consumo de restos óseos como alimento ofrecido, hábito frecuente en la población en estudio $(19 \%)$. Si bien el lento masticar de huesos duros (huesos de mamíferos) en el perro ayuda a disminuir la acumulación de cálculo dental, la mayoría de felinos mastica poco los huesos y los deglute rápidamente (40); considerando adicionalmente que en nuestro país el consumo de huesos es principalmente de pollo (hueso neumático y astilloso); por lo que podemos aseverar que estos constituyeron un factor importante en la mayor prevalencia de enfermedades dentales en el presente estudio.

La mayor tendencia a presentar grados más severos de enfermedad periodontal con relación a una mayor edad, como lo describen algunos autores $(41,42)$, se debe a la naturaleza progresiva de la enfermedad periodontal y a la probabilidad de que los propietarios no adviertan alteraciones supragingivales, lo que permite que exista una lesión significativa del periodonto con el tiempo.

La frecuencia de piezas dentales afectadas por la enfermedad periodontal fue mayor que en otros reportes (43). Los premolares son usualmente los más afectados debido a que las glándulas salivales (parótida y cigomática) desembocan cerca a estos, lo cual predispone a la organización de placa bacteriana (44).

La fractura dental (31\%) se observó únicamente en los dientes caninos y en una frecuencia superior a la de otros reportes, que señalan entre un 10 a $20 \%$ de ocurrencia $(36,38)$; lo que se puede explicar por el alto porcentaje de consumo de alimento mixto con hueso en comparación a la población de otros estudios. Esto explica, además, la elevada presentación de placa dental en estos dientes por la acumulación de restos óseos y el trauma asociado al consumo de los mismos. Por último, no se determinó la frecuencia de lesiones por reabsorción, toda vez que se requiere estudios radiográficos para el diagnóstico definitivo.

\section{CONCLUSIONES}

La enfermedad periodontal afecta al $95,5 \%$ de los felinos de los distritos del Cono Norte de Lima.

Existe un alto consumo de restos óseos como alimento ofrecido a los felinos (19\%).

Existe una alta prevalencia de fractura dental de los dientes caninos $(31,0 \%)$, especialmente en animales mayores de 5 años $(\mathrm{p}<0,05)$.

\section{Correspondencia}

Ricardo Grandez

Correo electrónico: ricardo.grandez@upch.pe 


\section{REFERENCIAS BIBLIOGRAFICAS}

1. Reichart PA, Durr UM, Triadan H, Vickendey G. Periodontal disease in the domestic cat. A histophatology study. J Periodont Res.1964; 19: 6775.

2. Cox ER, Lepine AJ. New technologies for investigating dental issues. In: Recent advances in dental health management. Chap. 2. Lewisburg, Ohio USA: Iams Co.; 2003. p. 16-22.

3. Mills A. Oro-dental disease in cats. A feline practitioner-ss perspective. Vet Clin N Am-Small 1992; 22: 1297-1307.

4. Hennet P. Anatomía y fisiología dentales de los pequeños carnívoros. En: Crossley DA, Penman S (eds). Manual de odontología en pequeños animales. España: Harcourt. 1999. p. 129-141.

5. Carmichael DT. Feline dental problems. DVM Newsmagazine. 2004; 35: 12S-15S.

6. Niemec BA. Oral pathology. Top Companion Anim Med. 2008; 23(2): 59-71.

7. Bellows J. The practice of veterinary dentistry: A team effort. Iowa: Ames Iowa State University 1999. p. 165-182.

8. Brannan R. Feline periodontal disease: Diagnosis, treatment and prevention. Orlando, Florida: Proc North American Veterinary Conference; 2006.

9. Lonsdale T. Periodontal disease and leucopenia. J Small Anim Pract. 1993; 36: 542-546.

10. Gorrel C. Home care: products and techniques. Clin Tech Small Animal Pract. 2000; 15: 226-231.

Harvey CE. Management of periodontal disease: understanding the options. Vet Clin N Am-Small. 2005; 35: 819-836.

11. Pedersen NC. Inflammatory oral cavity diseases of the cat. Vet Clin N Am-Small. 1992; 22: 1337-1341.

Gorrel C. Feline oral cavity disease. Rhodes, Greece: XXIX World Congress of the World Small Animal Veterinary Association; 2004.

12. Manfra MS. Orofaringe. En: Manual clínico de pequeñas especies. México: McGraw-Hill Interamericana; 1996. p. 729-747.

13. Holmstrom S.E. Manejo periodontal. Selecciones Veterinarias. 1998; 6(5): 404-409.

14. Caifa AM. Periodontitis and its association with systemic disease. Guaruja, SP, Brazil: X World Veterinary Dental Congress; 2007.

15. Niemec BA. Periodontal disease. Top Companion Anim Med. 2008; 23: 72-80.

16. Harvey CE, Flax BM. Feline oral-dental radiographic examination and interpretation. Vet Clin N Am-17. Small. 1992; 22: 1279-1295.

17. Remeeus P. Odontología restauradora en pequeños carnívoros. En: Crossley DA, Penman S (eds). Manual de odontología en pequeños animales. España: Harcourt 1999.p. 207-227.
18. Healey KA, Dawson S, Burrow R, et al. Prevalence of feline chronic gingivo-stomatitis in first opinion veterinary practice. J Feline Med Surg. 2007; 9(5): 373-381.

19. Harvey CE. Patología oral felina. Diagnóstico y tratamiento. En: Crossley CA, Penman S (eds). Manual de odontología en pequeños animales. España: Ed. Harcourt 1999. p. 175-187.

20. Ingham KE，Gorrel C, Blackburn J, Farnsworth W. Prevalence of odontoclastic resorptive lesions in a population of clinically healthy cats. J Small Anim Pract. 2001; 42: 439-443.

21. Lommer MJ, Verstraete FJ. Prevalence of dental resorptive lesions in Swedish cats. J Vet Dent. 2003; 20: $140-142$.

22. Okuda A, Harvey CE. Etiopathogenesis of feline dental resorptive lesions. Vet Clin N Am-Small. 1992; 22: $1385-1404$

23. Holmstrom SE. Odontología restauradora. En: Holmstrom S. Técnicas dentales en perros y gatos. $2^{\text {da }}$ ed. México: McGraw-Hill Interamericana 2000.p. 348-350.

24. Birchard S, Carothers M. Aggressive surgery in the management of oral neoplasia. Vet Clin N Am-Small. 1990; 20: 1117-1140.

25. Herring ES, Smith MM, Roberston JL. Lymph node staging of oral and maxillofacial neoplasms in 31 dogs and cats. J Vet Dent. 2002; 19: 122-126.

26. Northrup NC, Selting KA, Rassnick KM, et al. Outcomes of cats with oral tumors treated with mandibulectomy: 42 cases. J Am Anim Hosp Assoc. 2006: 42: 350-360.

27. Bertone ER, Snyder LA, Moore AS. Environmental and lifestyle risk factors for oral squamous cell carcinoma in domestic cats. J Vet Intem Med. 2003; 17: 557-562.

28. Hayes AM, Adams VJ, Scase TJ, Murphy S. Survival of 54 cats with oral squamous cell carcinoma in United Kingdom general practice. J Small Anim Pract. 2007; 48: 394-399.

29. Stebbins KE, Morse CC, Goldschmidt MH. Feline oral neoplasia: a ten-year survey. Vet Pathol. 1089; 26: 121-128.

30. Manfra MS. Recognition and treatment of oral tumors. Atlantic City, New Jersey: Atlantic Coast Veterinary Conference; 2001.

31. Robinson J, Gorrel C. Exploración y radiografías orales. En: Crossley DA, Penman S (eds). Manual de odontología en pequeños animales. España: Harcourt 1999. p. 43-62.

32. Bonagura JD. Kirk terapéutica veterinaria de pequeños animales. Vol II. España: McGraw-Hill Interamericana 2001. p. 1321-1346.

33. Wayne WD. Bioestadística: base para el análisis de las ciencias de la salud. $4^{\text {ta }}$ ed. México: Editorial Limusa; 2005. p. 755 p. 
34. Verhaert L, Van Wetter C. Survey of oral diseases in cats in Flanders. Vlaams Diergen Tijds. 2004; 73: 331-334.

35. Holmstrom SE. Profilaxis dental. En: Técnicas dentales en perros y gatos. $2^{\text {da. }}$ ed. México: McGrawHill Interamericana 2000; p 133-166.

36. Poblete CMS. Descripción y caracterización de las patologías dentales más frecuentes en felinos domésticos. Tesis de Médico Veterinario Zootecnista. Valdivia, Chile. Universidad Austral de Chile, 2006; 45 pp.

37. Gawor JP, Reiter AM, Jodkowska K, Kurski G, Wojtacki MP, Kurek A. Influence of diet on oral health in cats and dogs. J Nutr. 2006; 136: 2021S-2023S.

38. Case LP, Carey DP, Hirakawa A, Daristotle L. Salud dental y dieta. En: Case LP, Carey DP. Hirakawa A, Daristotle L (eds). Nutrición canina y felina. Guía para profesionales de los animales de compañía. $2^{\text {da }}$ ed. España: Harcourt Brace 2001. p. 477-488.
39. Manfra MS. Periodontal disease in dogs and cats. Atlantic City, New Jersey: Atlantic Coast Veterinary Conference; 2001.

40. Flornaes MLT, Lund M, Kortegaard HE, Arnbjerg J. Forekomst af parodontitis og FORL hos perserkatte. Dansk Veterinaertidsskrift. 2007; 90(11): 24-29.

41. Samsar E, Besalti Omer, Ozak A. Diagnostic approach to the prevalence of feline periodontal disease. Turk J Vet Anim Sci. 2003; 27: 349-357.

42. Radostits O, Mayhew IG, Houston DM. Exploración clínica del aparato digestivo. En: Radostits O, Mayhew IG, Houston DM (eds). Examen y diagnóstico clínico en Veterinaria. España: Harcourt; 2002. p. $349-359$.

Recibido: 01/08/2013

Aceptado: 21/11/2013 